\title{
A Novel Online Cleaning System for Shell and Tube Oil Heat Exchanger
}

\author{
Su Yan, Zhengyu Cao, Meirong Lian, Yingfan Liu, Jinhui Zhu ${ }^{\mathrm{a}}$ \\ CNOOC Energy Conservation \& Environmental Protection Service Co., Ltd., Tianjin 300452,

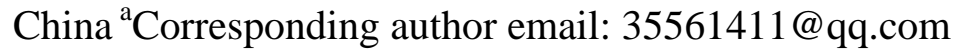

Keywords: Online; Cleaning system; Oil; Shell and tube heat exchanger

\begin{abstract}
Cleaning problem is extremely important to heat exchangers in industry such as crude oil processing systems because fouling can not only bring the reduction of heat transfer efficiency, but also affect the safety of the system. Common cleaning technologies used in shell and tube heat exchangers such as condensers of power plant include mechanical and chemical methods. This paper introduces a novel online cleaning system for shell and tube crude oil heat exchangers, which can work at the temperature as high as 500 degrees Celsius. It can be used especially in the crude oil processing system to remove the fouling without any interrupt of the working system.
\end{abstract}

\section{Introduction}

Heat exchangers are quite common devices in many industry process. Fouling is the accumulation of unwanted deposits on the heat transfer surfaces of a heat exchanger and is a major unresolved problem after the invention of heat exchangers. The serious financial and performance consequences of these problems have raised the profile of heat exchanger fouling as an important area of study. The worldwide costs, associated specifically with crude oil fouling in preheat trains were equal to around $20 \%$ of all heat exchanger fouling which is estimated to be of the order of $\$ 4.5$ million/year [1-2]. Many studies have been conducted in this regard and many techniques have been developed to evaluate and reduce fouling. W. Ebert et al. [3] introduced the theoretical concepts to the phenomenon of fouling. They modelled the fouling process using a rate equation and introduced the concept of threshold temperature below which fouling is minimum. F. Coletti et al. [4] developed a dynamic mathematical model which is capable of describing tube-side crude oil fouling in shell and tube heat exchangers as a function of local conditions. This model is able to devise a procedure to systematically analyze plant data and estimate necessary model parameters using primary plant measurements such as temperatures and flow rates. Mariusz Markowski et al. [5] presented a novel method for online determination of the thermal resistance of fouling in shell and tube heat exchangers. It can be applied under the condition that the data on pressure, temperature, mass flowrate and thermophysical properties of both heat-exchanging media are continuously available. Fouling of a heat exchanger is studied mainly to understand the mechanism of fouling process and to protect the heat exchanging surface so that an un-interrupted operation of the heat exchanger can be achieved without remarkable degradation in its performance [6]. Dillip Kumar Mohanty et al. [7-8] used C-factor as a tool for investigation of the performance of a heat exchanger due to fouling which consequently gives information regarding the extent of fouling developed on the heat transfer surfaces, and developed a local linear wavelet neural network based model to 
predict the temperature differences on both the tube and shell side and the heat exchanger efficiency. The aim of this paper is to present a novel online cleaning system for crude oil heat exchanger.

\section{Traditional Cleaning Methods}

\subsection{Artificial Chemistry Method}

In the world, including China, artificial chemistry is the most widely known and widely used method. However, there is a big drawback in this way. During the pressure test after the artificial chemical cleaning of the heat exchanger, leakage often occurs. This cannot achieve the effect of completely clear, and cannot reach the effective use of equipment and energy saving purposes. The use of chemical reagents not only increases the possibility of the unit to be scrapped and its reagent emission rises pollution problem to customers.

\subsection{Small Ball Cleaning Mechanical Method}

The small ball cleaning method is from Germany. Since the technology is relatively old and is not improved effectively, the function of this method cannot bring the expected cleaning effect. The cases of widespread utilization prove that the traditional ball cleaning method not only has low efficiency, but also increases a lot of safety risks to the system, because of the improper design on the structure of collecting net, which enlarges the water resistance of heat exchanger and causes ball wear and loss problem. Due to the principle of fluid mechanics, the cleaning rate cannot reach $100 \%$ of tubes, only 70-90\%. Furthermore, additional pump of this method should be running uninterruptedly, producing additional energy consumption, which reduces the cleaning effect. Energy saving efficiency can only reach 4-6\%, far less than expected.

\section{Online Cleaning Technology for Water Cooling System}

One online cleaning technology for water cooling system is automatic tube cleaning system (ATCS) explored by Israel CQM company. It keeps heat exchanger and condensers, using water as the working fluid, clean by running soft polymer balls through the tubes on preset time cycles, eliminating fouling and residue deposits and build-up without harmful chemicals. The soft balls slightly larger in diameter than the tubes rub them clean and are then trapped on the outlet of the heat exchanger where they are collected for the next cleaning cycle, as shown in Figure 1. 


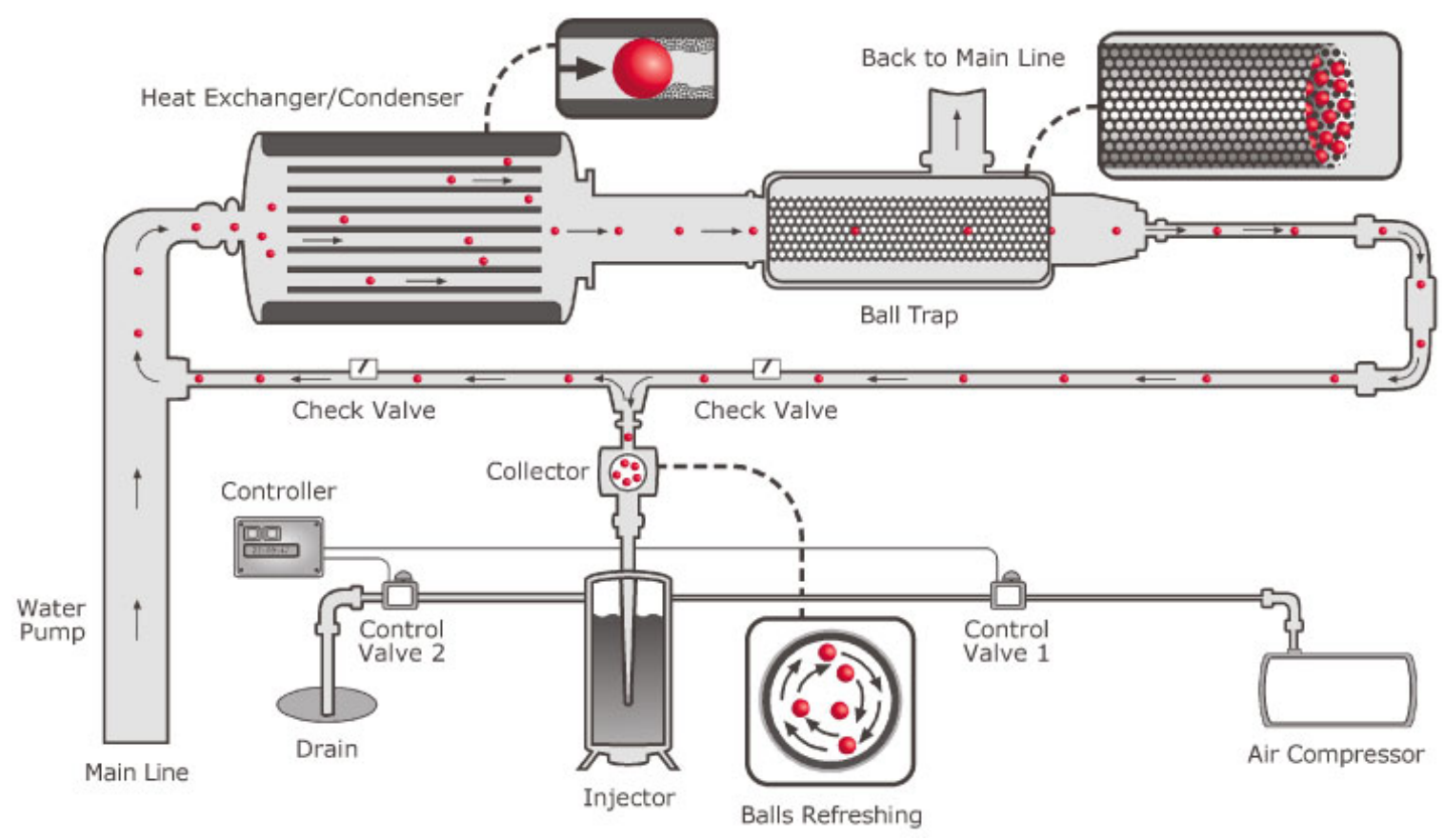

Figure 1. Schematic diagram of online cleaning system for water cooling heat exchanger

\section{Online Cleaning Technology for Crude Oil Processing System}

When the property of the treating fluid such as crude oil is not the same as water. The system needs to change especially the key component cleaning ball. One example is in the processing of petrochemicals, such as cracking of petrochemicals which is typically preformed at pressure of up to 220 atmospheres and temperatures of around 500 degrees Celsius. These temperatures prohibit the use of polymer balls of the type described above in water cooling system.

Figures 2 and 3 show the cleaning process and ball collecting process respectively. The ball is combined by stainless steel fiber and boron-silicon rubber, and this cleaning ball can endure high temperature as high as 500 degrees Celsius. In Figure 2, control valves $116 \mathrm{~b}$ and $116 \mathrm{~d}$ are pen and 116a and 116c are closed so that pump (114) carries crude oil from A to B, passing through the cleaning balls (102) storage room 110 to bring the balls. Then the cleaning balls go with crude oil enter into the tube of the heat exchanger (106). After the cleaning balls have passed through the heat exchanger and have been caught in trap (108) and there are two unidirectional check valves (112a and 112b) to employ the balls to keep the flow direction with crude oil. After cleaning, in Figure 3, control valves 116a and 116c are pen and 116b and 116d are closed. As a result, pump (114) draws liquid from trap retrieval port $C$ through storage room (110), thereby accumulating. The cleaning balls back into their storage location where they are stopped by a sieve arrangement. The flow from $\mathrm{C}$ is then returned to the primary flow path at $\mathrm{D}$. 


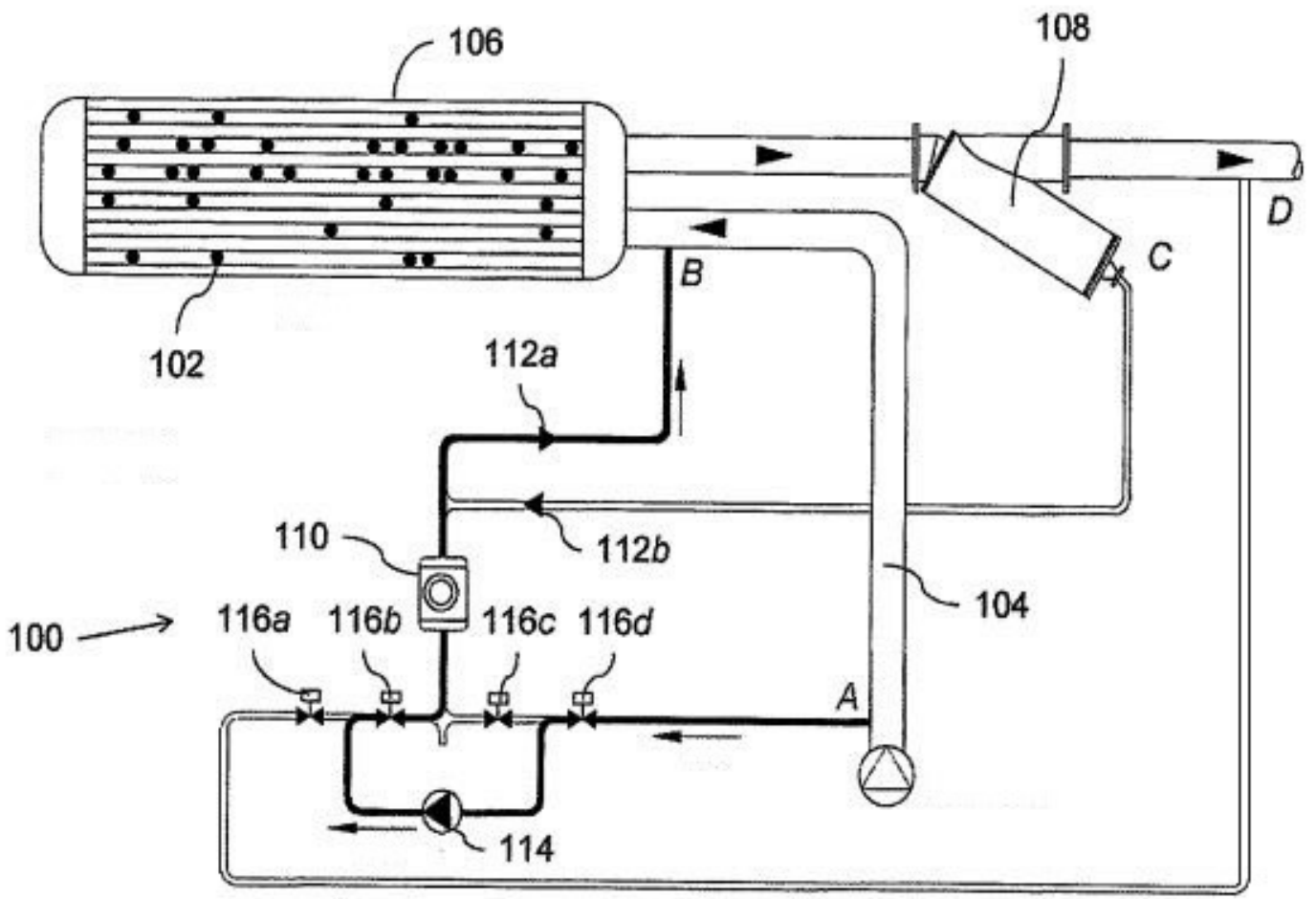

Figure 2. Online cleaning process for crude oil heat exchanger.

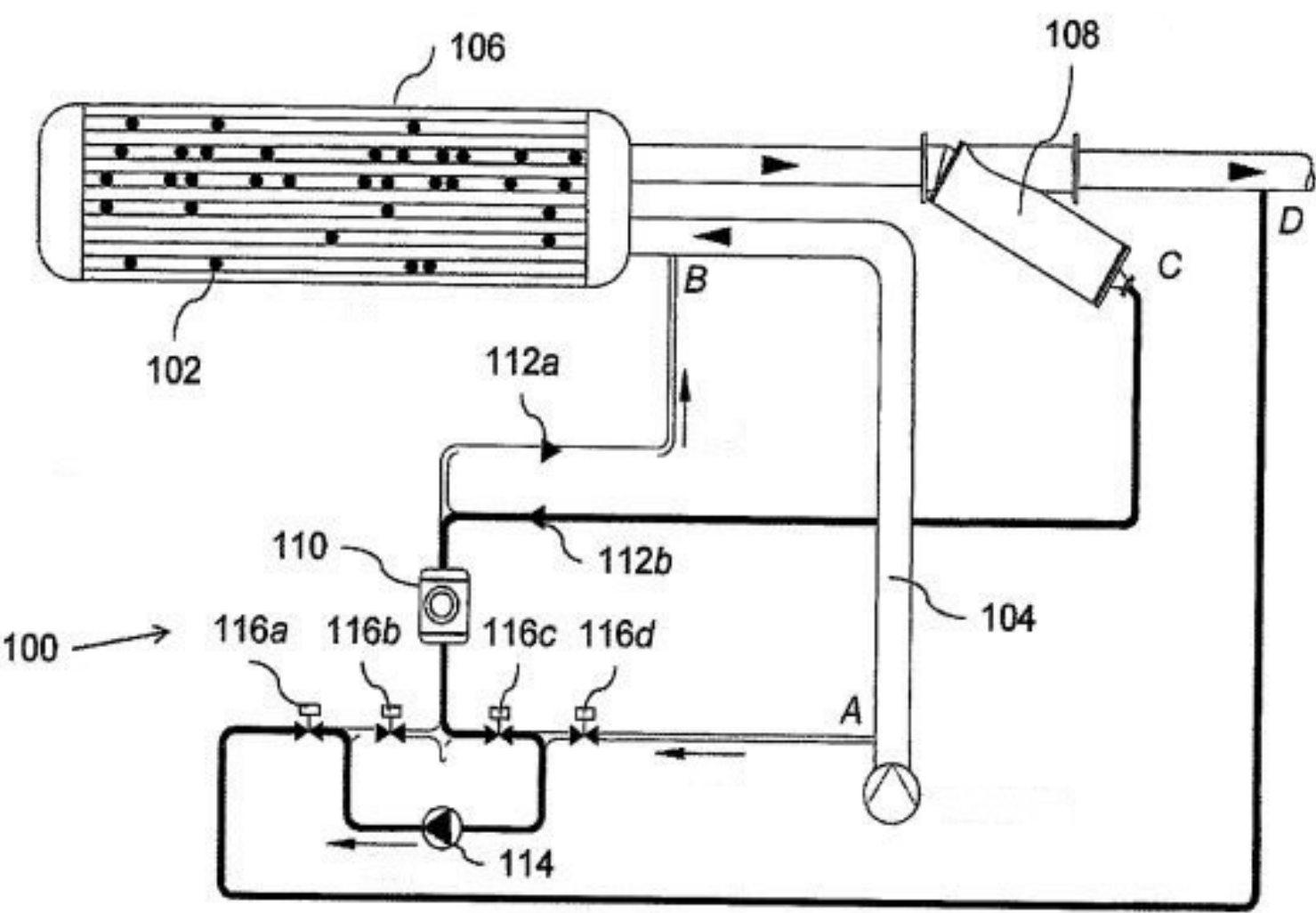

Figure 3. Ball collecting process after cleaning for crude oil heat exchanger. 


\section{Conclusion}

In this work, a novel online cleaning system for crude oil heat exchanger is presented and compared with another cleaning technology for water cooling system. The key component of the new system is using stainless steel fiber combined with boron-silicon rubber, which can improve the high temperature performance. This is a very promising technology in the crude oil processing industry.

\section{Acknowledgements}

This work was financially supported by the International S\&T Cooperation Program of China (No. 2013DFG42440).

\section{References}

[1] J. Aminian, S. Shahhosseini. Evaluation of ANN modeling for prediction of crude oil fouling behaviour. Applied Thermal Engineering, 2008, 28, 668-674.

[2] Dillip Kumar Mohanty, Pravin M. Singru. Fouling analysis of a shell and tube heat exchanger using local linear wavelet neural network. International Journal of Heat and Mass Transfer, 2014, 77, 946-955.

[3] W. Ebert, C.B. Panchal. Analysis of exxon crude-oil-slip-stream coking data, in: Proceedings of Fouling Mitigation of Industrial Heat-Exchanger Equipment, California, USA, 1995.

[4] F. Coletti, S. Macchietto. Distributed model of shell-and-tube heat exchangers undergoing crude oil fouling. Industrial \& Engineering Chemistry Research. 2011, 50, 4515-4533.

[5] Mariusz Markowski, Marian Trafczynski, Krzysztof Urbaniec. Validation of the method for determination of the thermal resistance of fouling in shell and tube heat exchangers. Energy Conversion and Management, 2013, 76, 307-313.

[6] Lalot S. On-line detection of fouling in a water circulating temperature controller (WCTC) used in injection moulding part 1: principles. Applied Thermal Engineering, 2006, 26, 1087-1094.

[7] Dillip Kumar Mohanty, Pravin M. Singru. Use of C-factor for monitoring of fouling in a shell and tube heat exchanger. Energy, 2011, 36, 2899-2904.

[8] Dillip Kumar Mohanty, Pravin M. Singru. Fouling analysis of a shell and tube heat exchanger using local linear wavelet neural network. International Journal of Heat and Mass Transfer, 2014, 77, 946-955. 